East African Medical Journal Vol. 80 No. 1 January 2003

VITAMIN A SUPPLEMENTATION ON CHILD MORBIDITY

J. Haidar, MD, MSc, D. Tsegaye, MSc, Ethiopian Health and Nutrition Research Institute, Addis Ababa, Ethiopia, D.H. Mariam, MD, PhD, Department of Community Health, Addis Ababa University, P.O. Box 11950, Addis Ababa, Ethiopia, H.N. Tibeb, MD, MPH, Health, Population and Nutrition Section, USAID office, Addis Ababa, Ethiopia and N.M. Muroki, BSc, MSc, PhD, MPS, Applied Human Nutrition Programme, University of Nairobi, P.O. Box 442, Uthiru, Nairobi, Kenya

Request for reprints to: Dr. J. Haidar, Ethiopian Health and Nutrition Research Institute, P.O. Box 5654, Addis Ababa, Ethiopia

\title{
VITAMIN A SUPPLEMENTATION ON CHILD MORBIDITY
}

\section{J. HAIDAR, D. TSEGAYE, D.H. MARIAM, H.N. TIBEB and N.M. MUROKI}

\begin{abstract}
Objective: To determine the impact of vitamin A supplementation on child morbidity and nutritional status.

Design: A community based follow-up (interventional) in nature.

Setting: Two randomly selected Weredas (districts) of Tigray, North Ethiopia were studied between 1996 and 1997.

Subjects: Four thousand seven hundred and seventy children aged between six and 72 months, selected using a multi-stage sampling procedure were enrolled and clinically assessed for xerophthalmia and nutritional status. A sub-sample of these children $(n=281)$ was further assessed for their serum retinol levels.

Main outcome measures: The pre and post intervention data on xerophthalmia, morbidity, nutritional status and serum retinol levels were compared.

Results: Vitamin A capsule coverage of $87 \%$ in all the villages of the Weredas and a statistically significant $(p<0.05)$ reduction in the prevalence of Bitot's spot (from 1.5 to 0.5 ), fever (from 29.8 to 14.2), diarrhoea (from 30.2 to 18.2), oedema (from 9.2 to 3.2), measles (from 14.0 to 6.2), conjunctivitis (from 10.2 to 3.0), stunted (from 64.2 to 42.7), wasted (from 12.8 to 2.5) and underweight (from 46.2 to 24.2). The proportion of children with normal serum retinol concentration $(>0.7 \mu$ mole/ $L$ ) has also improved significantly (from 36.8 to 56.2).

Conclusion: In conclusion, the significant improvement in morbidity and nutritional status that followed the intervention programme although encouraging, it still indicates the importance of coupling periodic provision of Vitamin A capsules with nutrition education.
\end{abstract}

\section{INTRODUCTION}

Vitamin A deficiency (VAD) is a global public health problem affecting populations in more than seventy-five developing countries, where clinical and sub clinical conditions have been observed(1). It is one of the major causes of childhood morbidity in economically deprived countries. As it is associated with impaired growth failure and high incidence of infections, it also contributes to increased childhood mortality(2-3). Evidence now shows that the survival chances of pre-school children are increased when vitamin A status is improved through biannual distribution of mega dose capsules(4).

In Ethiopia, several studies have established the fact that Vitamin A deficiency is a major public health problem(5-8). A more recent study conducted in three regions of rural Ethiopia has also shown Vitamin A deficiency to be a serious public health problem even in areas where previously low prevalence rates have been reported(9). Cognizant of the problem in the nation, an attempt was made by the government to supplement mega dose vitamin A capsule through the existing health infrastructure of the Ministry of Health since 1998. Although the supplementation programme was disease targeted, universal supplementation was also attempted in Arsi region, south west of the capital, where VAD emerged as an epidemic in 1995. Based on the lessons learned from these trials of 1995, the Ministry of Health adopted a universal vitamin A supplementation strategy in most parts of the country among which one of the areas was Tigray region. The newly adopted strategy was a biannual provision of 200,000 IU for all children aged between one and five years, and a single dose of 100,000 IU for all children below one year, with the objective of reducing the incidence of VAD and VAD associated morbidities in the country.

In order to evaluate the impact of the adopted intervention programme, and get a more complete and reliable estimation on the prevalence of vitamin $\mathrm{A}$ deficiency, vitamin A capsule coverage, morbidity and nutritional status in randomly selected Weredas of the region, biological and ecological indicators were collected before and after the implementation of the programme.

The pre-intervention survey was conducted in May/ 
June 1996, in a total of 5253 children aged between six and 72 months were examined for xerophthalmia and assessed for their health and nutritional status and serum retinol levels $(\mathrm{n}=247)$. The results showed that vitamin A deficiency and malnutrition was of major public health problem.

In this study, pre and post intervention data on morbidity and malnutrition for the 1997 survey in the Tigray region is presented to assist institutions working towards the elimination of Vitamin A deficiency in Ethiopia in implementing or monitoring and evaluation of intervention programmes.

\section{MATERIALS AND METHODS}

This was a cross-sectional follow-up study which made use of constructed comparison of baseline data to evaluate the one year relative impact of the universal vitamin A capsule supplementation programme in two randomly selected Weredas (districts) of Tigray region between 1996 and 1997. The region is located $780 \mathrm{kms}$ north of the capital city. The climate in the region is dry and agriculturally poor. The main crops grown in the area are sorghum, tef (Eragrostis tef), maize, and barley. The two study Weredas were $60 \mathrm{kms}$ from the centre of the region.

The size of the sample for clinical examination was defined with the objective of comparing xerophthalmia status in the study groups before and after programme implementation. Assuming a $1 \%$ average prevalence of Bitot's spot, the intervention was designed to test a $50 \%$ reduction in the incidence of xerophthalmia with $80 \%$ power of demonstrating a significant difference at $5 \%$ level. This gave a sample size of 5,684 (2842 per Wereda) in the two study Weredas.

Informed oral consent was obtained from the mothers/care takers of all the children participating in the study after the nature and the objectives of the study were clearly explained to them. Data were then collected on vitamin A status and coverage, morbidity and nutritional status from all the children using a pretested structured questionnaire, and a sub-sample of these children were further assessed for their serum retinol concentration levels.

A total of 4770 children $(83.9 \%$ of the required sample size), aged between six and 72 months were examined for xerophthalmia in accordance with WHO classification for xerophthalmia by trained physicians(10-11), and assessed for morbidity, nutritional status and vitamin A capsule coverage; while a sub-sample of these children $(n=281)$ were further assessed for serum retinol level.

For all anthropometrics measurements, children wore light clothing and were barefoot. Weights of children were measured to the nearest $0.1 \mathrm{~kg}$ with a portable beam balance, calibrated at regular intervals. Length and height measurements were made to the nearest centimetre with a locally made wooden board with fixed footplate and separate headboard. Recumbent length was measured for children aged above 24 months, and height was measured for older children; height-for-age, weight-for- age, and weight-for-height $\mathrm{z}$ scores (HAZ, WAZ, and WHZ, respectively) were derived by using the National Centre for Health Statistics (NCHS) reference standards (12-13), for determining nutritional status. The results were classified as stunting, underweight or wasting if they had height for age, weight for age and weight for height scores - 2 SD below the corresponding NCHS reference.
Blood samples for retinol analysis were taken from the ante-cubital vein using vaccutainer system from every 20 th child selected systematically. The collected blood was centrifuged at ambient temperature in dark room and the clear serum was pipetted into cryo-vials and stored at $-20^{\circ} \mathrm{C}$ until transported to South Africa (University of Stellenbosch) for serum retinol analysis. Serum retinol was analysed in a room illuminated with yellow light by an isocratic HPLC method of Bieri et al(14). The HPLC instrumentation was a Varian Liquid Chromatograph (model 5000, Varian Associates Inc., Palo Alto, CA). An $\mathrm{C}_{18}$-reverse phase column ( $\mu$ Bondapack) was used (4x125mm with $5 \mu \mathrm{m}$ particle size) An C $18 \mu$ Bondapack guard column was attached to the primary column. Sample preparation was by following the method of Driskell et al.(15). The internal standard solution, which contained all-trans-retinyl acetate in absolute ethanol, was added to the serum. An aliquot was injected into a column using a Rheodyne (model 7161; Rheodyne, Cottai, CA) with a $20 \mu \mathrm{L}$ sample loop and eluted at ambient temperature with an isocratic solvent, HPLC grade methanol: water $(95: 5 \mathrm{v} / \mathrm{v})$ at a constant flow rate of $2.0 \mathrm{ml} / \mathrm{min}$. Absorbance was monitored at $285 \mathrm{~nm}$ with a Variable Wavelength Detector (model, Varian Associates Inc., Palo Alto, CA). A Varian integrator (model 4290, Varian Associates Inc., Palo Alto, CA) was used to calculate peak areas. Retinol was quantified by comparing the peak area of retinol with that of retinyl acetate internal standard peak area assayed simultaneously(14). Normal limits of serum retinol concentrations were established according to the criteria described by WHO and Van der Beg(16-17), which considered concentration $>0.7$ micro- mole per litre, $<0.7$ and 0.35 micromole per litre as normal, indicative of moderate risk, and high risk of deficiency respectively.

Data were analysed for changes in morbidity, nutritional status and serum retinol levels using the Statistical Package for Social Sciences (SPSS) and ANTHRO softwares. To compare the difference before and after the intervention of the programme a Chi square and t-tests were used for dichotomous and continuous outcomes respectively.

\section{RESULTS}

Overall coverage of vitamin A capsule in all the villages during the one-year period was $87 \%$. The pre and post intervention distribution of clinical and sub-clinical xerophthalmia is shown in Table 1 . The prevalence of night blindness $(\mathrm{XN})$ slightly changed from $0.9 \%$ to $0.8 \%$, which may imply that the ongoing intervention was not significant when $\mathrm{XN}$ is considered $(\mathrm{P}>0.05)$. On the other hand, the reduction in the incidence of Bitot's spot's, which was three folds reduction, was highly significant $(\mathrm{P}<0.01)$.

The proportion of children with normal levels of serum retinol concentration of children substantially increased from $36.8 \%$ to $56.2 \%$, which is nearly three fold (Table 1); while the proportion of children with moderate risk reduced from $16.2 \%$ to $6.8 \%$ (about three times) children with high risk of deficiency reduced from $47.0 \%$ to $37.0 \%$ (about 1.5 times). When compared with the baseline data, the proportion of children with normal values of serum retinol concentration, the improvement achieved after the intervention was significantly higher than the proportion at baseline $(\mathrm{p}=0.01)$. This finding is 
supported by the significant improvement in the mean values of serum retinol concentration for the Weredas $(0.80 \pm 0.31, p=0.0001)$. There was no sex difference observed in retinal concentration among the children (data not shown).
The proportion of malnutrition according to the three malnutrition indices is shown in Table 2. The proportion of malnutrition at the baseline was significantly higher than after intervention. ( $\mathrm{p}<0.05$ for stunting, $\mathrm{p}<0.01$ for wasting and $\mathrm{p}<0.01$ for underweight).

Table 1

Prevalence of xerophthalmia before and after intervention (\%)

\begin{tabular}{lcccc}
\hline Characteristic & Before & After & SE & CI (95\%) \\
\hline Clinical & $($ No. $=5253)$ & $($ No. $=4770)$ & 0.001 & $-0.004-0.002^{\text {ns }}$ \\
$\quad$ Night blindness & 0.8 & 0.9 & 0.001 & $0.004-0.014^{* *}$ \\
$\quad$ Bitot's spot & 1.5 & 0.5 & & \\
Biochemical & & & & \\
(SRC) & No. $=247)$ & No. $=281)$ & & $0.02-0.16^{* *}$ \\
$\quad$ Deficient & 16.2 & 6.8 & 0.027 & $0.01-0.18^{*}$ \\
$\quad$ Low & 47.0 & 37.0 & 0.042 & $-0.30-0.08^{* *}$ \\
\hline
\end{tabular}

$\mathrm{SE}=$ Standard error.; NS = Not significant SRC $=$ Serum retinal level in $\mu$ mole $/ \mathrm{L}$

*Significant at 0.05

** Significant at 0.01

Table 2

Distribution of nutritional status before and after intervention (\%)

\begin{tabular}{lcccc}
\hline Nutritional status & Before & After & SE & CI (95\%) \\
\hline Stunted & 64.2 & 42.7 & 0.42 & $0.13-0.30^{*}$ \\
Wasted & 12.8 & 2.5 & 0.02 & $0.04-0.16^{* *}$ \\
Underweight & 46.2 & 24.2 & 0.04 & $0.11-0.32^{* *}$ \\
\hline
\end{tabular}

Total number of children examined before and after intervention were 5253 and 4770 respectively;

$\mathrm{SE}=$ Standard error

*Significant at 0.05

** Significant at 0.01

Table 3

Selected morbidity status before and after the intervention (\%)

\begin{tabular}{lcccc}
\hline Type of morbidities & Before & After & SE & CI (95\%) \\
\hline Diarrhoea & 30.2 & 18.2 & 0.03 & $0.04-0.14^{*}$ \\
Cough & 28.1 & 26.5 & 0.03 & $-0.02-0.12^{\text {ns }}$ \\
Fever & 29.8 & 14.2 & 0.3 & $0.64-0.25^{* *}$ \\
Measles & 14.0 & 6.2 & 0.02 & $0.02-0.13^{*}$ \\
Conjunctivitis & 10.2 & 3.0 & 0.02 & $0.01-0.12^{* *}$ \\
\hline
\end{tabular}

Total numbers of children examined before and after intervention were 5253 and 4770 respectively;

$\mathrm{SE}=$ Standard error

* Significant at 0.05

**Significant at 0.01 
Table 3 shows the proportions of children with different types of selected illnesses after intervention. There was significant reduction of the following morbidity incidences: fever ( $p<0.01)$, diarrhoea $(\mathrm{p}<0.05)$, measles $(\mathrm{p}<0.05)$ and conjunctivitis $(\mathrm{p}<0.01)$. However, reduction in the proportion of cases of cough $(p>0.5)$ was not statistically significant.

\section{DISCUSSION}

According to $\mathrm{WHO}$, the evaluation criteria that make it possible to state whether VAD is a public health problem are $1 \%$ for night blindness and $0.5 \%$ for Bitot's spot(16). In the present study, the post intervention figure obtained for night blindness remained practically the same as the baseline result. Although, history of night blindness has been reported to be a valid index of vitamin A deficiency among pre-school children(11), and potentially useful in screening xerophthalmia, its usefulness is questionable because of its subjectivity and misconception of the symptoms especially in developing countries. This raises a question on its usefulness of xerophthalmia for screening, particularly in evaluative studies(8). Given the limitations in use night blindness and since the prevalence of Bitot's spot's is more VAD specific, more reliable and easy to standardize, the discussion will be limited to the results obtained for Bitot's spot's, serum retinol values, nutritional and selected morbidity status.

Several studies conducted in developing countries on the impacts of periodic vitamin A supplementation have shown significant reduction in the prevalence of Bitot's spot's and an improvement in the serum retinol concentration(19). This is in conformity with study results in the present study. Even though, the three-fold improvement achieved in this study is encouraging, the level of Bitot's spot's of $0.5 \%$ in the region is still just at the WHO cut-off point. This indicates that the ongoing supplementation programme needs to be strengthened further with a nutrition education programme for its sustainability.

Malnutrition is probably the most important health problem both because of the long term effects it can have on intellectual and physical development, and because it is the underlying factor causing a great deal of morbidity and mortality(20). The findings that there was significant improvement in the nutritional indices after vitamin A supplementation support those of other researchers. Associations between anthropometric and xerophthalmia deficit have also been reported in children although are difficult to interpret probably due to confounding factors such as low dietary vitamin A intake, low protein and energy consumption, poor socio-economic status, and vulnerability to infections that inhibit growth(21).

The degree of VAD is proportional to the degree of growth retardation, stunting and frequent wasting(22). It also reported to that periodic large dose vitamin A supplementation has significant impact on growth in children with xerophthalmia(21). Similarly, studies in India(23) and $\mathrm{Nepal(24)}$ and Indonesia(25) reported improved growth with Vitamin A supplementation. The results indicate that vitamin A supplementation has significant effect in reducing child morbidity due to diarrhoea, fever, conjunctivitis and measles are in conformity with studies by other researchers(26). However, the study has failed to show a similar effect on cough. This is in conformity with findings by Dowell et al.(26). Despite the observation made by the two studies, further work is needed to explain the differences.

Cross-sectional on studies of impaired vitamin A status and various infections conducted in many developing countries have showed strong association between VAD and severe, chronic, persistent diarrhoea $(22,24)$.

In conclusion the coverage of VAD during the oneyear period $(87 \%)$ shows a relatively better achievement when compared to the Cambodian experience(27). It is likely that it has substantially reduced the prevalence of clinical and sub-clinical vitamin A deficiency. The data has also clearly indicated an improvement in morbidity and nutritional status. Periodic Vitamin A supplementation can therefore, together with nutrition education be recommended for sustainable supplementation programme.

\section{ACKNOWLEDGEMENTS}

To UNICEF-Ethiopia for sponsoring this study. We thank Dr. HailMichael G/Sillasie, the former EHNRI Deputy Director, Dr. Mira Shakar (UNICEF-Ethiopia) and the Tigray Regional Health Bureau in particular, Dr. Mesfin Minas and his colleagues for their unlimited assistance and facilitating our study. Finally, the assistance rendered by Professor D. Labadarious and Dr. I. Moodie (Stellenbosch university) is highly acknowledged.

\section{REFERENCES}

1. Mc Laren, D.S. Malnutrition and the eye. 1962; 172-179, 213214. Academic Press, New York.

2. Sommer, A. Incidence, prevalence and scale of blinding malnutrition. Lancet. 1986; 1:1407-1408.

3. Sommer, A., Tarwotjo, I., Hussain, G. and Susanto, D. Increased mortality in children with mild vitamin A deficiency. Lancet. 1983; 2:585-589.

4. Beaton, G.H. et al. Effectiveness of vitamin A supplementation in control of young child morbidity and mortality in the control of young child morbidity and mortality in developing countries. ACC/SCN Nutrition policy paper. Geneva. United nations administrative committee on coordination /Sub-committee on nutrition. 1993.

5. Wold-Gebriel, Z., Demeke, T. and West, C.E. Xerophthalmia in Ethiopia: a nation-wide ophthalmological, biochemical and anthropometric survey. Eur. J. Clin. Nutr. 1991; 45:469-478.

6. Haidar, J., Demissie, T., G/Sillasie, G., Fufa, H., Birratu, E. and Neka, T.H. Vitamin A deficiency status in Tigray region. Ethiop. J. Health Dev. 1999; 13:87-91.

7. Haidar, J. and Tibeb, H.N. Xerophthalmia in children of Torbayo Village, West Hararghe.Ethiop. J. Health Dev. 1998; 11:545-548. 
8. De Sole, G., Belay, Y. and Zegeye, B. Vitamin A deficiency in southern Ethiopia. Am. J. Clin. Nutr. 1987; 45:780-784.

9. Haidar, J. and Demissie, T. Malnutrition and xerophthalmia in rural communities of Ethiopia. East Afr. Med. J. 1999; 10: 590-593

10. Sommer, A. Vitamin A deficiency and its consequences. A field guide to detection and control. WHO, Geneva, 3rd. 1995; 69.

11. Sommer, A., Hussaini, G., Muhilal, H. et al. History of night blindness; a simple tool for xerophthalmia screening. Am. J. Clin. Nutr . 1980; 33:887-891.

12. Hamil, P.V., Drizd, T.A., Johnson, C.L. et al. Physical growth: National Centre for Health Statistics percentiles. Am. J. Clin. Nutr. 1979; 32:607-629.

13. WHO Working Group. Use and interpretation of anthropometric indicators of nutritional status. Bull. WId. Hlth. Org. 1986; 64:929-941.

14. Bieri, J.G., Tolliver. T.J. and Catigani, G.L. Simultaneous determination of $\alpha$-tocopherol and retinol in plasma or red blood cells by HPLC. Am J Clin Nutr. 1979 32:2143-2149.

15. Driskell, W.J., Bashor, M.M. and Neese, J.W. Loss of vitamin A in long-term stored, frozen sera. Clinica Chimica Acta. 1985; 147:25-35.

16. WHO. Control of vitamin A deficiency and xerophthalmia. WHO Technical Report Series, 1982; 672.

17. Van der Beg, H. Vitamin A intake and status. Eur. J. Clin. Nutr. 1996; 50:57-12

18. WHO. Control of vitamin A deficiency and xerophthalmia. WHO Technical Report series. 1982; 672.
19. Keith, P., West, J. and Sommer, A. Delivery of oral doses of vitamin A deficiency and nutritional blindness. ACC/SCN states of the art series, nutritional policy discussion paper number. 1987; 2.

20. Donal, S.M. and Martin, F. Sight and life manual on vitamin A deficiency disorder, $2^{\text {nd }}$ edition. 2000; 163.

21. Sommer, A. Nutritional blindness: Xerophthalmia and keratomalacia. Oxford University Press, New York. 1982.

22. Sommer, A. and West, K.P. Jr. Vitamin A deficiency: health, survival and vision. Oxford University Press, New York. 1996.

23. Bahl, R., Bhandar, N., Taneja, S. et al. The impact of vitamin A supplementation on physical growth of children is dependent on season. Eur. J. Clin. Nutr. 1997; 51:26-29.

24. West, K.P., Le Clerq, S.C., Shresta, S.R. et al . Effects of vitamin A on growth of vitamin A deficient children: Field studies in Nepal. J. Nutr. 1997; 127:1957-1965.

25. Hadi, H., Stoltzfus, J.R., Dibley, M.J. et al. Vitamin A supplementation selectively improves the linear growth of Indonesian preschool children: results from a randomized controlled trial. Am. J. Clin. Nutr. 2000; 71:507-513.

26. Dowell, S.F., Papic, Z., Bresee, J.S. et al . Treatment of respiratory syncitial virus infection with vitamin A: a randomized placebo controlled study trial in Santiago, Chile. Pediatr. Infet. Dis. J. 1996; 15:782-786.

27. Helen, K.I. The need for increasing coverage of vitamin A capsule programme to reduce vitamin A deficiency among young children in Cambodian. Sight and life newsletter. 2000; 4 . 